\title{
Review of Adamantinoma Tumor Characteristics and Treatments in the National Cancer Database (NCDB/ NCD)
}

\author{
McMahon KM ${ }^{*}$, Nilles-Melchert TT $^{1}$, Shonnard \\ $\mathbf{M J}^{1}$ and Silberstein $\mathbf{P J}^{2}$ \\ ${ }^{1}$ Medical Student, Creighton University School of \\ Medicine, USA \\ ${ }^{2}$ Professor, Creighton University School of Medicine, USA \\ *Corresponding author: McMahon KM, Medical \\ Student, Creighton University School of Medicine, USA
}

Received: August 13, 2021; Accepted: September 15, 2021; Published: September 22, 2021

\begin{abstract}
Background: Adamantinomas are rare low-grade malignant bone tumors of uncertain origin, which manifest primarily in the anterior mid-tibial shaft. Growth is indolent with significant risk of delayed-onset recurrence and/or distant metastasis. The aim of this study is to describe the physical characteristics and treatments of adamantinomas in patients diagnosed through the NCDB.
\end{abstract}

Methods: This is a retrospective study of all patients diagnosed with adamantinomas between 2004 and 2017 in the National Cancer Database. Adamantinomas were identified using ICD-O-3 code 9261. Description statistics and univariate Kaplan-Meier curves were performed using SPSS. No patients were excluded from analysis.

Results: $97 \%$ of adamantinoma lesions were in the long bones of the lower extremities.

Incidence was similar bilaterally, behavior was universally invasive, grade was $66.1 \%$ undetermined, stage was $63.7 \%$ stage 1 , and mean diameter was $63.67 \pm 42.8 \mathrm{~mm}$. Only 2 instances of metastasis (bone) at diagnosis were recorded. Treatment was overwhelmingly surgery with an open approach radical excision or resection of the primary lesion with limb salvage and clean surgical margins regardless of tumor grade, stage, primary site, or size. 5, 10, and overall survival were $96.8 \%, 94.2 \%$, and $92.9 \%$ respectively.

Conclusion: Adamantinomas are rare slow-growing malignant tumors that primarily affect the anterior cortex of the tibia and have significant potential for recurrence and metastasis, thus should be treated with radical surgical resection and excision to obtain clear margins. Overall prognosis is favorable, but patients should be enrolled in a long-term surveillance program to periodically rule-out recurrence or metastasis.

Keywords: Adamantinoma; Sarcoma; Tumor characteristics; Treatment; Survival

\section{Abbreviations}

NCDB/NCD: National Cancer Database; SEER: Surveillance, Epidemiology and End Results; CT: Computed Tomography; MRI Magnetic Resonance Imaging; ICD-O: International Classification of Diseases for Oncology; PUF: Participant User Files; NOS: Not Otherwise Specified; R-LS: Radical Excision/Resection with Limb Salvage; AD: Classic Adamantinomas; OFD-AD: Osteofibrous Dysplasia-like (Differentiated) Adamantinomas; OFD: Osteofibrous Dysplasia

\section{Introduction}

Adamantinomas are rare low-grade malignant bone tumors that primarily affect the anterior tibia [1-5]. Their origin is uncertain but is believed to be epithelial due to their biphasic malignant epithelial and benign osteolytic presentation on biopsy [1-3,6-8]. The mean age of onset is 25-35 years (range 2-86 years) and there is a slight preponderance for male sex $[6,7,9,10]$. Overall mortality is favorable as a recent study by Aytenik et al. (2020), reported a 10-year survival rate of $91.5 \%$ [7].

Clinically adamantinomas present with dull pain, swelling, erythema, or a mass, but also may less frequently present with bowing deformities or paraneoplastic syndromes [1,4,5]. Diagnosis is primarily via histology $[1,2,11]$, but CT or MRI imaging may help detect spread or medullary involvement $[2,5,8,11]$. Due to their malignant invasive nature and ability to metastasis years-decades after treatment, treatment is primarily radical limb salvage surgery or amputation to obtain clear margins $[1,5,12]$.

The purpose of this study is to review the treatment and tumor characteristics of adamantinomas in literature and to analyze characteristics as recorded in the NCDB. This is to the authors' best knowledge the largest study conducted on adamantinoma tumor characteristics and treatments and the first one conducted through the NCDB.

\section{Methods}

A retrospective review of adamantinoma cases was conducted
Sarcoma Res Int - Volume 6 Issue 1 - 2021

Submit your Manuscript | www.austinpublishinggroup.com

McMahon et al. () All rights are reserved
Citation: McMahon KM, Nilles-Melchert TT, Shonnard MJ and Silberstein PJ. Review of Adamantinoma Tumor Characteristics and Treatments in the National Cancer Database (NCDB/NCD). Sarcoma Res Int. 2021; 6(1): 1049. 
using the most recent data available through the National Cancer Database (NCDB) Participant User Files (PUF) for bone and joint tumors (Published in 2017). The 2017 PUF contains data collected on patients diagnosed between 2004 and 2017. The NCDB was formed to improve patient outcomes globally and now contains approximately $70 \%$ of all new patients in the United States diagnosed with cancer. Professional registrars enter the individual level de-identified data from accredited Commission on Cancer facilities (American College of Surgeons certified hospitals) [13].

Patients with adamantinomas were identified using ICD-O-3 (International Classification of Diseases for Oncology, Third Edition) histology code 9261. All patients recorded in the bone and joint tumors PUF with this histological tag were included in this study, 168 in total.

All data was analyzed using SPSS statistical software version 27.1. Data was analyzed for frequencies of diagnostic confirmation method, surgical and diagnostic staging procedure, primary tumor site, laterality, behavior, grade, stage, metastasis, lymphovascular invasion, tumor size, surgical procedure, surgical approach, surgical margins, regional lymph node surgery, surgery at non-primary site, 30-day readmission, radiation therapy, chemotherapy, and last contact or all-cause mortality months from diagnosis. Means and/or median were likewise calculated for tumor size, delay until surgical diagnostic and staging procedure, delay until $1^{\text {st }}$ surgical procedure, delay until definitive surgical, duration of post-surgical inpatient stay, and last contact or all-cause mortality months from diagnosis. Crosstabs were used to compare trends in tumor grade, stage, size, and primary site vs surgical approach, surgical procedure, and surgical margins. Kaplan Meier Curves were conducted to evaluate the effects of the above listed factors on patient mortality.

Days to first surgical procedure and days to definitive surgical procedure were grouped into 3 categories of 0-24 days, 25-49 days, $50+$ days. Tumor size was divided into 4 groups (1-32 mm, 33-64 $\mathrm{mm}, 65-97 \mathrm{~mm}, \& 98+\mathrm{mm}$ ) which were each approximately $1 / 2$ of the mean diameter. Inpatient stay was divided into 2 groups (less than 1 week and $1+$ weeks).

41 cases of adamantinomas were reported as having a diameter of $999 \mathrm{~mm}$. The authors determined this was likely a data input error due to the presence of a similar NCDB variable (tumor size summary vs tumor size) that listed unknown tumor diameter as 999. Thus, these cases were filtered from analysis. After removal of these cases, 3 cases reporting diameters of 989,800 , and $420 \mathrm{~mm}$ were identified as extreme outliers via box-and-whisker plot analysis. These 3 cases were likewise removed from analysis.

\section{Results}

\section{Overview \& diagnosis}

168 cases of adamantinomas were diagnosed in the NCDB, 165 cases $(98.2 \%)$ were diagnostically confirmed by histology, 2 cases (1.2\%) by cytology, \& 1 case $(0.6 \%)$ by radiographic examination. Surgical and diagnostic staging procedure was reported in 168 cases and was performed after a mean of 6.21 days (range 0-361 days). The types of procedures reported were 49 cases (29.2\%) without surgical diagnostic or staging procedure performed, 2 cases (1.2\%) with a biopsy performed at a non-primary site without exploratory

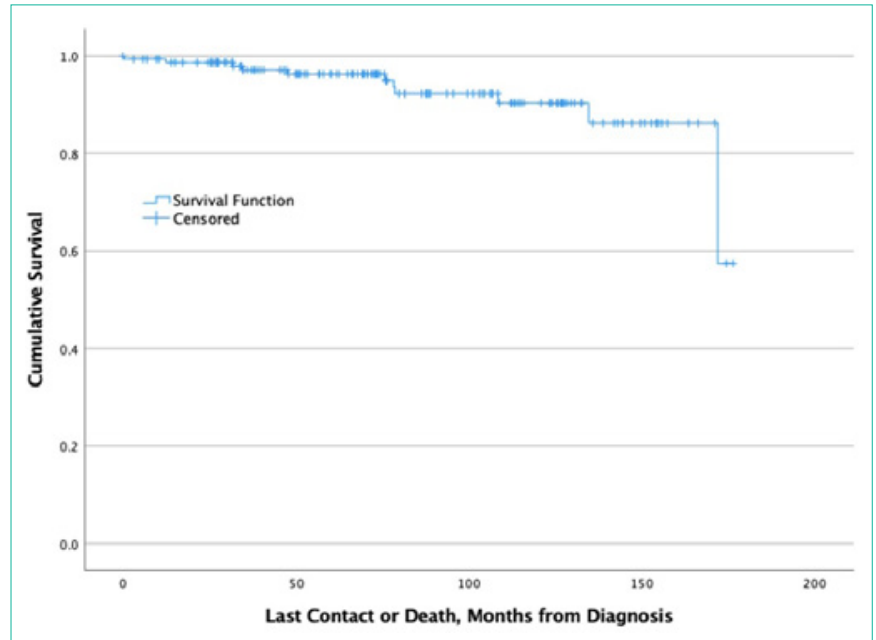

Figure 1: Overall mortality (Kaplan-Meier Curve).

procedure, 114 cases $(67.9 \%)$ with a biopsy of the primary site, 1 case $(0.6 \%)$ with a surgical procedure with bypass but without biopsy, 1 case $(0.6 \%)$ reported an exploratory procedure with biopsy of the primary or non-primary site, and 1 case $(0.6 \%)$ reported no information on whether a diagnostic or staging procedure was performed.

\section{Tumor characteristics \& surgical treatment}

85 cases $(50.6 \%)$ were lateralized to the right side of the body, 81 cases $(47.6 \%)$ to the left, and 2 cases $(1.2 \%)$ were unspecified. Accounting for outliers, tumor size (diameter) was reported in 124 cases with a mean of $63.67 \pm 42.761 \mathrm{~mm}$, a median $55.00 \mathrm{~mm}$, and a range of $10-220 \mathrm{~mm}$. All 168 cases were reported as having invasive behavior. Grade, stage, primary site, and diameter are recorded and compared to surgical approach, surgical procedure, and surgical margins in Table 1-3. Surgical treatment for adamantinomas regardless of tumor grade, stage, primary site, or size was primarily an open approach with radical excision and resection with limb salvage and clear surgical margins. All limb amputations were performed on lower limb tumors and are listed in Table 4.

The mean number of days before the $1^{\text {st }}$ surgical procedure or the definitive surgical procedure was 52.01 days (range 0-1096 days) and 59.36 days (range 0-1096 days) respectively. Mean duration of post-surgical inpatient stay was 4.57 days (range 0-47 days) and 3 cases $(1.8 \%)$ of unplanned and 4 cases $(2.4 \%)$ of planned readmission within 30 days after surgical discharge were reported.

Metastasis at diagnosis was reported in 66 cases, there was bone metastasis in 2 cases (3\%), but no reported metastasis to the brain, liver, lungs, distant lymph nodes or other sites. No lymphovascular invasion of the primary tumor site was reported.

No cases of regional lymph node surgery were recorded. 1 case of surgical resection at a non-primary site was reported and 2 cases reported regional resection. 11 cases (6.5\%) reported that surgery was not performed because it was not part of the first treatment course and 1 case $(0.6 \%)$ reported that surgery was recommended but refused by the patient, their family, or a legal guardian.

\section{Tumor treatment (Non-surgical)}

Only one instance of radiation therapy was reported in all 168 
Table 1: Surgical procedure by tumor grade, stage, primary site, and diameter.

\begin{tabular}{|c|c|c|c|c|c|c|c|}
\hline Characteristic (\#) & $\begin{array}{c}\text { No surgery } \\
(13)\end{array}$ & $\begin{array}{l}\text { Local Excision } \\
\text { (12) }\end{array}$ & $\begin{array}{c}\text { Partial } \\
\text { Resection (16) }\end{array}$ & $\begin{array}{c}\text { Radical Excision or } \\
\text { Resection with limb salvage } \\
(112)\end{array}$ & $\begin{array}{l}\text { Amputation } \\
\text { NOS (2) }\end{array}$ & $\begin{array}{c}\text { Partial } \\
\text { Amputation (9) }\end{array}$ & $\begin{array}{c}\text { Full } \\
\text { amputation (1) }\end{array}$ \\
\hline \multicolumn{8}{|l|}{ Grade (165) } \\
\hline $1(39)$ & 3 & 3 & 7 & 24 & 0 & 2 & 0 \\
\hline $2(15)$ & 0 & 1 & 1 & 12 & 1 & 0 & 0 \\
\hline $3(2)$ & 1 & 0 & 0 & 1 & 0 & 0 & 0 \\
\hline $4(1)$ & 0 & 0 & 0 & 1 & 0 & 0 & 0 \\
\hline Undetermined (108) & 9 & 8 & 8 & 74 & 1 & 7 & 1 \\
\hline \multicolumn{8}{|l|}{ Stage (165) } \\
\hline $1(107)$ & 4 & 5 & 11 & 78 & 2 & 6 & 1 \\
\hline $2(4)$ & 1 & 0 & 0 & 3 & 0 & 0 & 0 \\
\hline $3(2)$ & 0 & 0 & 1 & 0 & 0 & 1 & 0 \\
\hline Undetermined (48) & 6 & 7 & 4 & 29 & 0 & 2 & 0 \\
\hline \multicolumn{8}{|l|}{ Primary site (165) } \\
\hline Upper Limb (5) & 1 & 1 & 0 & 3 & 0 & 0 & 0 \\
\hline Lower Limb (160) & 12 & 11 & 16 & 109 & 2 & 9 & 1 \\
\hline \multicolumn{8}{|l|}{ Diameter } \\
\hline 1-32 mm (34) & 2 & 2 & 4 & 25 & 0 & 0 & 1 \\
\hline 33-64 mm (35) & 2 & 3 & 5 & 24 & 0 & 1 & 0 \\
\hline $65-97 \mathrm{~mm}$ (31) & 0 & 2 & 2 & 23 & 1 & 3 & 0 \\
\hline $98+m m(65)$ & 9 & 5 & 5 & 40 & 1 & 5 & 1 \\
\hline
\end{tabular}

Table 2: Surgical approach by tumor grade, stage, primary site, and diameter.

\begin{tabular}{|c|c|c|c|}
\hline Characteristic (\#) & No surgery (10) & $\begin{array}{l}\text { Robotic converted } \\
\text { to open (1) }\end{array}$ & Open $(80)$ \\
\hline \multicolumn{4}{|l|}{ Grade (91) } \\
\hline $1(26)$ & 3 & 0 & 23 \\
\hline $2(10)$ & 0 & 0 & 10 \\
\hline $3(1)$ & 1 & 0 & 0 \\
\hline $4(1)$ & 0 & 0 & 1 \\
\hline Undetermined (53) & 6 & 1 & 46 \\
\hline \multicolumn{4}{|l|}{ Stage (91) } \\
\hline $1(74)$ & 4 & 1 & 69 \\
\hline $2(2)$ & 1 & 0 & 1 \\
\hline $4(3)$ & 2 & 0 & 1 \\
\hline Undetermined (12) & 3 & 0 & 9 \\
\hline \multicolumn{4}{|l|}{ Primary site (91) } \\
\hline Upper Limb (2) & 0 & 0 & 2 \\
\hline Lower Limb (89) & 10 & 1 & 78 \\
\hline \multicolumn{4}{|l|}{ Diameter (91) } \\
\hline 1-32 mm (16) & 2 & 1 & 13 \\
\hline 33-64 mm (14) & 1 & 0 & 13 \\
\hline $65-97 \mathrm{~mm}(18)$ & 0 & 0 & 18 \\
\hline $98+\mathrm{mm}(43)$ & 7 & 1 & 36 \\
\hline
\end{tabular}

cases. It was reported that therapy was performed on an upper limb lesion 234 days after diagnosis and lasted 42 days. 30 fractions of
phase-I radiation were administered via external beam NOS with a total phase dose of 5400 centigray. The tumor was of undetermined grade and size but was categorized as stage 4 . Surgical status was unknown.

Only 2 instances of chemotherapy were reported. Both cases reported that multidrug chemotherapy agents were given in the $1^{\text {st }}$ course of therapy before surgical procedure of the site was performed. These tumors were stage 1 lower limb lesions of undetermined grade and diameters of 58 and $115 \mathrm{~mm}$ respectively.

No other instances of hormone, immunotherapy, or hematologic transplant or endocrine therapies were administered or reported.

\section{Tumor Treatment (Outcomes)}

Last contact or all-cause mortality months from diagnosis was reported in 155 cases and had a mean follow-up of $80.39 \pm 45.378$ months (range 0-176 months). Mortality at 5 years, 10 years, and overall follow-up was $3.2 \%, 5.8 \%$, and $7.1 \%$ with 5,9 , and 11 reported deaths in this period. Kaplan-Meier curves were run for primary site, laterality, grade, stage, bone metastasis, tumor diameter, days until first surgical procedure, days until definitive surgical procedure, surgical procedure, surgical approach, surgical margins, post-surgical inpatients stay duration, and 30-day hospital readmission. All variables had 155 cases for follow-up data except bone metastasis which had 66, days to $1^{\text {st }}$ surgical procedure and pays to definitive surgical procedure which had 139, surgical procedure which had 143, surgical approach which had 77, and post-surgical inpatients stay duration which had 135 (Table 5). Census of each patient characteristic are listed in the left-most column of Table 5. Mortality was independent of all factors 
Table 3: Tumor margins by tumor grade, stage, primary site, and diameter.

\begin{tabular}{|c|c|c|c|c|}
\hline Characteristic (\#) & No residual tumor (131) & Residual tumor NOS (4) & Microscopic Residual Tumor (5) & Indeterminate margins (9) \\
\hline \multicolumn{5}{|l|}{ Grade (148) } \\
\hline $1(35)$ & 31 & 2 & 1 & 1 \\
\hline $2(14)$ & 12 & 1 & 1 & 0 \\
\hline $3(1)$ & 1 & 0 & 0 & 0 \\
\hline $4(1)$ & 1 & 0 & 0 & 0 \\
\hline Undetermined (97) & 86 & 1 & 3 & 7 \\
\hline \multicolumn{5}{|l|}{ Stage (148) } \\
\hline $1(98)$ & 90 & 2 & 4 & 2 \\
\hline $2(3)$ & 3 & 0 & 0 & 0 \\
\hline $3(2)$ & 2 & 0 & 0 & 0 \\
\hline $4(2)$ & 2 & 0 & 0 & 0 \\
\hline Undetermined (43) & 34 & 2 & 1 & 6 \\
\hline \multicolumn{5}{|l|}{ Primary site (148) } \\
\hline Upper Limb (3) & 2 & 0 & 0 & 1 \\
\hline Lower Limb (145) & 129 & 4 & 5 & 7 \\
\hline \multicolumn{5}{|l|}{ Diameter (148) } \\
\hline 1-32 mm (31) & 27 & 1 & 1 & 1 \\
\hline 33-64 mm (33) & 29 & 1 & 2 & 1 \\
\hline 65-97 mm (29) & 29 & 0 & 0 & 2 \\
\hline $98+\mathrm{mm}(55)$ & 46 & 2 & 2 & 3 \\
\hline
\end{tabular}

Table 4: Amputations and tumor diameter.

\begin{tabular}{|l|c|}
\hline Amputation type (\# of amputations) & Tumor Size (mm) \\
\hline Amputation NOS (2) & 80,110 \\
\hline Partial amputation (9) & $40,75,80,80,111,115,115,140,180$ \\
\hline Complete limb amputation (1) & 15 \\
\hline
\end{tabular}

listed in Table 5 besides tumor metastasis. Only tumor metastasis had significant correlation with decreased survivability.

\section{Discussion}

The purpose of this study was to characterize the anatomical and physiological characteristics of adamantinoma tumors and to review the methods used to treat those tumors reported within the NCDB. Due to the rarity of this tumor, there is little information currently available on these topics and no prior studies conducted through the NCDB.

In this paper, the main focus is on classic adamantinomas. One of the most important factors when diagnosing classic Adamantinomas (AD) is differentiating it from similar entities such as Osteofibrous Dysplasia-Like (Differentiated) Adamantinomas (OFD-AD) and osteofibrous dysplasia (OFD). Prior studies have theorized that these 3 conditions exist along a spectrum, but few cases have shown progression from one form to another and cases where this was observed may be due to misdiagnosis $[1,3,5,8,9,10,11]$. Classic adamantinomas are the most severe as they are malignant tumors with the greatest potential to recur and/or metastasize [3,5,8,9]. Osteofibrous dysplasia-like adamantinomas are of intermediate severity as they rarely metastasize $[3,5]$. Osteofibrous dysplasias are the most benign as they seldom progress in childhood and have not been shown to progress after puberty $[3,5,9,11]$.

These 3 conditions have similar symptoms (pain, swelling, bone deformity), location of lesion manifestation, cytogenetic irregularities, immunohistochemistry, histological appearance, and imaging findings $[1,3-5,9,11]$. However, they can be differentiated by their degree of epithelial cell involvement, tumor diameter, medullary involvement, imaging characteristics, and age of onset. $\mathrm{AD}$ has the greatest degree of epithelial involvement, is most likely to have more significant or complete invasion of the medullary cavity, has a greater tumor diameter, on imaging shows skip (patchy) lesions, has a more pronounced "moth-eaten" border, and most often presents in patients greater than 20 years of age $[1,3,8,9,11]$. OFD-AD has significantly less epithelial involvement on histology (may only be seen focally) and most often presents in patients less than 20 years of age $[1,3,8,9,11]$. OFD shows scant epithelial involvement and mostly present in patients less than 10 years of age $[3,5,8,9,11]$.

Differentiation of these conditions is important because the treatment for each is different. OFD often only requires observation and symptom management, but occasionally will require surgical intervention if the lesion compromises bone structural integrity. It is also recommended that surgical intervention wait until after puberty due to a $25 \%$ rate of recurrence following local resection or curettage in larger lesions if done before $[5,9,11]$. OFD-AD treatment is controversial and not well established as Most et al. (2010) reported that it was only recently diagnosed as a separate entity from classic adamantinomas [9]. They note that of the few reported cases of OFD-AD, no metastasis had been reported, thus some clinicians 
Table 5: Patient 5 year, 10-year, and overall survival by tumor characteristics \& treatment.

\begin{tabular}{|c|c|c|c|c|}
\hline Characteristics/treatment (census) & Log-Rank P-value & 5-year Survival (\%) & 10-year Survival (\%) & Overall Survival (\%) \\
\hline \multicolumn{5}{|l|}{ Primary Site (155) } \\
\hline Upper Limb (5) & & 100 & 100 & 100 \\
\hline Lower Limb (150) & 0.326 & 96.7 & 94 & 92.7 \\
\hline \multicolumn{5}{|l|}{ Laterality (155) } \\
\hline Right (78) & & 96.2 & 94.9 & 92.3 \\
\hline Left (75) & & 97.3 & 94.7 & 94.7 \\
\hline Unspecified (2) & 0.032 & 100 & 50 & ** \\
\hline \multicolumn{5}{|l|}{ Grade (155) } \\
\hline $1(36)$ & & 100 & 100 & 100 \\
\hline $2(13)$ & & 92.3 & 92.3 & 84.6 \\
\hline $3(1)$ & & 100 & 100 & ** \\
\hline $4(1)$ & & 100 & ** & ** \\
\hline Undetermined (104) & 0.352 & 96.2 & 92.3 & 91.3 \\
\hline \multicolumn{5}{|l|}{ Stage (155) } \\
\hline $1(96)$ & & 96.9 & 96.9 & 95.8 \\
\hline $2(3)$ & & 100 & 100 & ** \\
\hline $3(2)$ & & 100 & 100 & 100 \\
\hline $4(5)$ & & 80 & 80 & 60 \\
\hline Undetermined (49) & 0.303 & 98 & 89.8 & 89.8 \\
\hline \multicolumn{5}{|l|}{ Presence of Bone Metastasis (66) } \\
\hline No Metastasis (64) & & 96.9 & 96 & ** \\
\hline Distant Bone Metastasis (2) & $<0.001$ & 50 & 50 & ** \\
\hline \multicolumn{5}{|l|}{ Diameter (155) } \\
\hline 1-32 mm (32) & & 96.9 & 96.9 & 96.9 \\
\hline 33-64 mm (36) & & 97.2 & 91.7 & 91.7 \\
\hline $65-97$ mm (31) & & 96.8 & 93.5 & 87.1 \\
\hline $98+\mathrm{mm}(56)$ & 0.719 & 96.4 & 94.6 & 94.6 \\
\hline \multicolumn{5}{|l|}{ Days to 1st Surgical Procedure (139) } \\
\hline $0-24$ days $(55)$ & & 98.2 & 98.2 & 98.2 \\
\hline 25-49 days (49) & & 93.9 & 91.8 & 89.8 \\
\hline $50+$ days $(39)$ & 0.163 & 100 & 97.4 & 94.9 \\
\hline \multicolumn{5}{|l|}{ Days to Definitive Surgical Procedure (139) } \\
\hline $0-24$ days $(40)$ & & 97.5 & 97.5 & 97.5 \\
\hline 25-49 days (55) & & 94.5 & 93.7 & 90.9 \\
\hline $50+$ days $(48)$ & 0.356 & 100 & 97.9 & 95.8 \\
\hline \multicolumn{5}{|l|}{ Surgical Procedure (143) } \\
\hline No Surgery (10) & & 90 & 80 & 80 \\
\hline Local Excision (12) & & 91.7 & 83.3 & 83.3 \\
\hline Partial Resection (15) & & 100 & 100 & 100 \\
\hline R-LS (103) & & 98.1 & 96.1 & 94.2 \\
\hline Limb Amputation NOS (2) & & 100 & 100 & 100 \\
\hline Partial Limb Amputation (9) & & 88.9 & 88.9 & 88.9 \\
\hline Total Limb Amputation (1) & & 100 & 100 & ** \\
\hline Surgery NOS (2) & & 100 & 100 & 100 \\
\hline
\end{tabular}




\begin{tabular}{|c|c|c|c|c|}
\hline Unknown (1) & 0.629 & 100 & 100 & 100 \\
\hline \multicolumn{5}{|l|}{ Surgical Approach (77) } \\
\hline No Surgery (7) & & 85.7 & 85.7 & 85.7 \\
\hline Robotic converted to Open (1) & & 100 & 100 & 100 \\
\hline Open approach (70) & 0.092 & 97.1 & 97.1 & 97.1 \\
\hline \multicolumn{5}{|l|}{ Surgical Margins (155) } \\
\hline No residual tumor (122) & & 98.4 & 95.9 & 94.3 \\
\hline Residual tumor NOS (3) & & 100 & 100 & 100 \\
\hline Microscopic residual tumor (5) & & 80 & 80 & ** \\
\hline Margins not evaluable (8) & & 100 & 100 & 100 \\
\hline No primary site surgery (10) & & 90 & 80 & 80 \\
\hline Unknown/non-applicable (7) & 0.051 & 85.7 & 85.7 & 85.7 \\
\hline $0-6$ days $(110)$ & & 97.3 & 94.5 & 94.5 \\
\hline $7+$ days $(24)$ & 0.915 & 100 & 100 & 91.7 \\
\hline \multicolumn{5}{|l|}{ Hospital 30-day Readmission (155) } \\
\hline No readmission (144) & & 97.2 & 95.1 & 93.8 \\
\hline Unplanned readmission (2) & & 100 & 100 & 100 \\
\hline Planned readmission (4) & & 75 & 75 & 75 \\
\hline Unknown Readmission Status (5) & 0.817 & 80 & 80 & 80 \\
\hline
\end{tabular}

recommend observation of lesions with plans for wide surgical resection if the lesion progresses or if bone integrity is compromised $[3,5,9]$, but others recommend radical excision of OFD-AD to reduce risk of sampling errors and misdiagnosis $[8,9]$. $\mathrm{AD}$ is a malignant tumor with recurrent and metastatic potential; thus, it always requires surgery $[3,5,8,9,11]$.

Diagnosis of adamantinomas is primarily by histological confirmation with immunohistochemical staining due to the biphasic presentation of benign osteofibrous and malignant epithelial components on surgical tissue biopsy [1-3,6-8]. Epithelial cells have been shown to stain positive for keratins $5,14,17$, and 19, and vimentin, but not keratins 8 or 18 regardless of histological subtype $[1,3,6,10]$. Prior studies have reported that the periphery of classic adamantinomas may mimic the more benign OFD-AD, and OFD-AD may only show epithelial cells focally, therefore, large biopsies of the tumor core should be collected to maximize diagnostic accuracy and minimize the chance of misdiagnosis of similar conditions $[1,3,8,11]$. OFD-AD \& OFD show similar epithelial staining to AD but to a lesser degree [3]. Cytogenetics have also shown that adamantinomas, OFD$\mathrm{AD}$, and OFD often possess trisomy of chromosome $7,8,12,19,21$, or $22[1,3,9,10]$.

On imagining, these lesions tend to appear as longitudinally oriented lesions of the anterior mid-diaphyseal tibia. Adamantinomas form eccentric or central masses with singular or multifocal osteolytic lesions and intervening sclerotic rings, which give it a "soap bubble" appearance. The mass may also present with significant cortical destruction, medullary invasion, or soft-tissue involvement $[1,3,5,8,9,11]$. Staging and presurgical preparation of and for classic adamantinomas should utilize contrast-enhanced MRIs as multidirectional imaging of the affected bone may help identify tumor extension and/or distant foci that may be missed on radiographs. Intense contrast enhancement on T2-weighted MRIs shown in $\mathrm{AD}$ and OFD-AD may be due to high levels of osteofibrous stromal vascularity $[1,5,8,9,11]$.

In this study, all cases were diagnosed between 2004 and 2017 and all were reported as classic adamantinomas with invasive behavior. Tumors were also primarily low or undetermined grade and earlystage tumors that affected the long bones of the lower extremities. Other studies reported similar findings and have further localized these tumors to the anterior cortical tibial midshaft with occasional involvement of the bone marrow and ipsilateral fibula. Rarely, there is also involvement of other bones such as the humerus, femur, spine, ribs, carpals, or tarsals [1-3,5-12]. The mean tumor diameter in this study was $63.67 \mathrm{~mm}$ (range $10-220 \mathrm{~mm}$ ). This was dissimilar from the findings in Khanna et al. (2008) which retrospectively analyzed 24 patients with $\mathrm{OFD}$, OFD-AD, or $\mathrm{AD}$ that had biopsy confirmation and imaging. They reported an OFD mean diameter of $61 \mathrm{~mm}$ (range 20-85 mm), an OFD-AD mean diameter of $65 \mathrm{~mm}$ (range 20-130 $\mathrm{mm}$ ), and an $\mathrm{AD}$ mean diameter of $132 \mathrm{~mm}$ (range 65-260 mm) [11].

There is potential that some of the cases reported in the NCDB as classic adamantinomas were misdiagnosed osteofibrous-like adamantinomas since that was a newly acknowledged diagnosis within the data collection period for this study. The contrast in tumor diameter between this study and the Khanna et al. (2008) may also be due to misdiagnosis in this study or due to the larger sample size of this study showing a more accurate mean tumor diameter.

This study found that adamantinomas rarely have metastatic lesions at diagnosis, but other studies reported that untreated or undertreated tumors locally recur in $18-32 \%$ of cases or metastasize 
to regional lymph nodes, the lungs, or bony structures in $10-30 \%$ of cases, sometimes more than 10 years after the initial diagnosis and treatment $[1,3,4,7-9,11]$.

The most common treatment of adamantinoma tumors in this study regardless of grade, stage, primary site, or size was radical excision/resection with limb salvage via open approach, and with clear surgical margins. Due to the metastatic and recurrent potential of this tumor, it is reasonable that most surgical treatments were aggressive and had an open approach to obtain clear surgical margins. Prior studies report that adamantinoma treatment is almost universally aggressive surgical treatment with clean surgical margins to minimize risk of metastasis or recurrence [1,3,4,6-9,11,12]. After radical resection and excision, limb reconstruction may be performed with interventions such as allografts, autografts, metallic replacements, or distraction osteogenesis $[5,8,9,11,12]$.

This study did not find any statistically significant relationship between mortality and most of the factors listed in Table 5, but there was a significant relationship between mortality and tumor metastasis. Unfortunately, due to the paucity of cases (only 2 metastases reported), no strong conclusion can be drawn from these results. Prior studies on adamantinomas have suggested that metastasis does increase the risk of mortality $[9,12]$ and studies on other moreaggressive sarcomas such as osteosarcoma and Ewing sarcoma note that metastasis is a critical factor in patient prognosis $[14,15]$.

In this study, it was reported that 11 cases had no surgery of the primary site in the $1^{\text {st }}$ course of treatment. It is unknown why these 11 patients did not have surgery initially, but it may have been due to factors not listed in the NCDB such as socioeconomic disparities or ineligibility for surgery. It also may have been that they were found to have OFD-AD not requiring surgery, but this was not differentiated from $\mathrm{AD}$ in the $\mathrm{NCDB}$ reporting.

The invasive nature of this tumor and its ability to recur or metastasize years after therapy means that patients should be placed on long-term surveillance. Studies show that most local recurrences occur within 5-15 years but may occur years-decades beyond this range. Metastasis also may occur more than 10-20 years after surgical intervention $[5,6,11,12]$. The overall prognosis is favorable for all patient groups in this study, but because of the delayed onset of recurrence or metastasis, the follow-up period of $80.39 \pm 45.378$ months (0-176 months) may be insufficient to detect significant changes in mortality.

\section{Conclusion}

This is to the author's best knowledge the largest study on adamantinoma tumor characteristics and treatment, and the first one conducted through the NCDB. Adamantinomas are rare slowgrowing malignant tumors that primarily affect the anterior cortex of the tibia and have significant potential for delayed recurrence and metastasis. Thus, they should be treated with radical surgical resection and excision to obtain clear margins and minimize risk of recurrence and metastasis. Overall prognosis is favorable and is independent of most factors recorded in this study, but patients should be enrolled in a long-term surveillance program to periodically rule-out recurrence or metastasis, which could prove lethal if undetected and untreated.

\section{References}

1. Jain D, Jain V, Vasishta R, Prabhat Ranjan P, Kumar Y. Adamantinoma: A clinicopathological review and update. Diagnostic Pathology. 2008; 3.

2. Limaiem F, Tafti D, Malik A. Adamantinoma. StatPearls. Treasure Island (FL). StatPearls Publishing. 2021.

3. Kahn L. Adamantinoma, osteofibrous dysplasia and differentiated adamantinoma. Skeletal Radiology. 2003; 32: 245-258.

4. Kitsoulis P, Charchanti A, Paraskevas G, Marini A, Georgios Karatzias G. Adamantinoma. Acta Orthopaedica Belgica. 2007; 73: 425-431.

5. Rajani R. Osteofibrous Dysplasia and Adamantinoma. (AA Surgeons, Producer) Retrieved 2021, from orthoinfo.aaos.org.

6. Jundt G, Remberger K, Roessner A, Schulz A, Bohndorf K. Adamantinoma of Long Bones: A Histopathological and Immunohistochemical Study of 23 Cases. Pathology - Research and Practice. 1995; 191: 112-120.

7. Aytekin N, Öztürk R, Amer K. Epidemiological Study of Adamantinoma from US Surveillance, Epidemiology, and End Results Program: III Retrospective Analysis. Journal of Oncology. 2020; 1-8.

8. Van der Woude H, Hazelbag H, Bloem J, Taminiau A, Hogendoorn P. MRI of Adamantinoma of Long Bones in Correlation with Histopathology. American Journal of Roentgenology. 2003: 1737-1744

9. Most M, Franklin S, Inwards C. Osteofibrous Dysplasia and Adamantinoma Journal of American Academy of Orthopaedic Surgeons. 2012; 18: 358-366.

10. Ali N, Niada S, Morris M, Brini A, Huen D, Sumathi V. Comprehensive Molecular Characterization of Adamantinoma and OFD-like Adamantinoma Bone Tumors. The American Journal of Surgical Pathology. 2019; 43: 965974.

11. Khanna M, Delaney D, Tirabosco R, Sifuddin A. Osteofibrous Dysplasia, Osteofibrous Dysplasia-like Adamantinoma and Adamantinoma: Correlation of Radiological Imaging Features with Surgical Histology and Assessment of the use of Radiology in Contributing to Needle Biopsy Diagnosis. Skeletal Radiology. 2008; 37: 1077-1084.

12. Qureshi Ai, Shott S, Mallin B, Gitelis S. Current Trends in the Management of Adamantinoma of Long Bones an International Study. Journal of Bone \& Joint Surgery. 2000; 82: 1122

13. Bilimoria K, Stewart A, Winchester D, Ko C. The National Cancer Database: A Powerful Initiative to Improve Cancer Care in the United States. Annals of Surgical Oncology. 2008; 15: 683-690.

14. Riggi N, Suva M, Stamenkovic I. Ewing's Sarcoma. New England Journal of Medicine. 2021.

15. Mialou V, Philip T, Kalifa C, Perol D, Gentet JC, Marec-Berard P, et al. Metastatic osteosarcoma at diagnosis: prognostic factors and long-term outcome--the French pediatric experience. Cancer. 2005; 104: 1100-1109. 